\title{
Mimosa scabrella Benth. as Facilitator of Forest Successional Advance in the South of Brazil
}

\author{
Paula Iaschitzki Ferreira ${ }^{1}$ (D), Juliano Pereira Gomes ${ }^{2}$ (D), Lilian Iara Bet Stedille ${ }^{2}$ (D), \\ Roseli Lopes da Costa Bortoluzzi² (D), Adelar Mantovani² (D) \\ ${ }^{1}$ Instituto Federal de Educação, Ciência e Tecnologia de Santa Catarina (IFSC), Lages, SC, Brasil \\ ${ }^{2}$ Universidade do Estado de Santa Catarina (Udesc), Lages, SC, Brasil
}

\begin{abstract}
Pioneer species have the potential to colonize disturbed environments, contributing to the establishment of other species and driving the dynamics and advancement of the structure of forest communities. The aim of this study was to assess the evolution of floristic-structural composition in the regenerating stratum of communities formed in the Mimosa scabrella Benth. understory with different ages. The study was carried out in four municipalities located in the Santa Catarina State South Plateau, on communities with different successional ages. The plot method was applied to the survey of all arboreal individuals with height $\geq 10 \mathrm{~cm}$. The floristic-structural patterns found were compatible with the expected trend of Araucaria Forest successional dynamic, showing higher richness and abundance of regenerating individuals in the older understories.
\end{abstract}

Keywords: bracatinga, forest succession, natural regeneration, facilitation, Araucaria Forest. 


\section{INTRODUCTION}

Tree species contribute directly and indirectly to improvements in the ecosystem and accelerate biodiversity restoration (Elliott et al., 2000). The natural regeneration of native populations of pioneer species is an important way to restore the functionality of altered environments, especially where propagules provided by the landscape matrix are available. Some authors have emphasized conducting natural regeneration as one of the most promising alternatives due to ecological and economic aspects (Alvarenga et al., 2006).

Studying natural regeneration represents an important factor for analysing successive evolutionary communities (Melo \& Durigan, 2007), and may indicate the effectiveness of tree cover in forming habitat which is favourable for colonising new species (Kabakoff \& Chazdon, 1996). Haggar et al. (1997) observed that trees with high growth rates generally stimulate higher levels of regeneration in their understory. Chada et al. (2004) verified that reforestation with tree legumes proved to be effective in activating natural succession mechanisms, and after seven years, 50 species of 25 botanical families have already colonized the understory of the study area.

Mimosa scabrella Benth., popularly known as bracatinga, is a tree species belonging to the Fabaceae family. It is native and endemic to Brazil (Dutra \& Amorim, 2012), and represents an important function in the secondary succession of natural or anthropic clearings of Araucaria Forest, where it can form dense, almost pure nuclei (Reitz et al., 1978). Due to its high adaptability to the edaphic soil conditions of river banks and patches (Reitz et al., 1978), tolerance to physical soil conditions (Inoue et al., 1984), and also its high levels of interactions with micro-organisms of the soil, entomofauna and vertebrates Araucaria Forest, it is considered one of the main indicated species for environmental restoration programs (Reis \& Kageyama, 2003).

Considering the adaptive potential of M. scabrella to colonise altered areas, it is expected that the regeneration existing in forest understories of populations of this species with different ages presents floristic-structural variations, following the patterns of forest succession dynamics. Thus, the objective was to evaluate the successional evolution of the regenerating arboreal stratum in understories of $M$. scabrella populations with different ages using analysis of floristic-structural patterns. Specifically, we sought to answer the following questions: (1) Are there variations in the diversity and richness of the regenerating stratum occurring in $M$. scabrella populations with different ages? (2) Does the floristic-structural pattern of the different $M$. scabrella understories reflect successional dynamics according to ecological groups?

\section{MATERIALS AND METHODS}

The study was conducted in an understory with natural Mimosa scabrella populations present in Montana Araucaria Forest remnants (IBGE, 2012) located in the Santa Catarina State South Plateau. Three populations were selected to cover different ages, which were defined by the succession time (natural regeneration) of the areas (Table 1). The average precipitation of the studied areas is $1,200 \mathrm{~mm} /$ year and the climate is $\mathrm{Cfb}$ by the Köppen classification (Alvares et al., 2014). The plots were located in gently undulating relief areas in the study sites.

Table 1. Description of the regenerating community areas in understory Mimosa scabrella Benth. populations, Santa Catarina State South Plateau.

\begin{tabular}{lccc}
\multirow{2}{*}{ Characteristics } & \multicolumn{2}{c}{ Mimosa scabrella Benth. populations } \\
\cline { 2 - 4 } & Four years & Seven years & Nine years \\
Municipality & Bocaina do Sul & Ponte Alta & Lages \\
Altitude $(\mathrm{m})$ & 850 & 880 & 916 \\
Mean temperature $\left({ }^{\circ} \mathrm{C}\right)$ & 16.5 & 16.9 & 16.5 \\
Soil type & Aluminic Cambisol & Humic Cambisol & Lithic Neosol \\
Total area of population $(\mathrm{ha})$ & 389.7 & 88.0 & 13.4 \\
\hline
\end{tabular}


The populations of four and seven years characterized areas destined to enlarging the riparian strips in silvicultural farms (Pinus and Eucalyptus genera). In the landscape context, the population of four years was located in a matrix with few conserved fragments, differing from the population of seven years (Figure 1).

The population of nine years colonised a natural environment after the last Merostachys multiramea Hack. reproductive event, occurring between 2006 and 2008 in the Santa Catarina mountain range (Santos et al., 2012). This area is located amid secondary forest fragments in medium-advanced successional stage and silvicultural plantations (Pinus spp.).

The regenerating shrub-arboreal vegetation in the understory of the M. scabrella populations was evaluated using the fixed plot method, two sample units per population, with dimensions of $40 \times 20 \mathrm{~m}$, totalling $800 \mathrm{~m}^{2} /$ population. All individuals with height $\geq 10 \mathrm{~cm}$ were sampled, with diameter-at-breast-height (DBH) measurements taken for individuals with $\mathrm{DBH} \geq 5 \mathrm{~cm}$, and collar diameter $(\mathrm{CD})$ for those with $\mathrm{DBH}<5 \mathrm{~cm}$.

For the floristic composition, all the species present in the sample units were identified in the field when possible, and botanical material was collected for those not identified for later identification in the LUSC Herbarium of the Santa Catarina State University.

The binomial nomenclature was verified using the List of Flora species of Brazil (Flora do Brasil 2020). Estimation and comparison of the richness among the study sites was performed by species/individual analysis using the rarefaction method with 1000 randomizations, generated based on the abundance data matrix in each sample unit. The Shannon diversity index $\left(\mathrm{H}^{\prime}\right)$ and the Pielou evenness index $\left(J^{\prime}\right)$ were calculated for estimating floristic diversity, which enable representing the distribution uniformity of individuals among the existing species. The $H^{\prime}$ value was compared between the areas using the Hutcheson t-test. A number of authors were consulted for characterising the species in relation to the ecological groups, using the data in works covering forests of the Atlantic Forest domain, especially consultations with Flora Ilustrada Catarinense (Reitz, 1971), where the nomenclature proposed by Budowski (1965) of pioneers (PI), early secondary (ES) and late secondary (LS) species were incorporated. The relative participation of the ecological groups (proportion of the individuals belonging to each ecological group in relation to the total sampled) for the three evaluated understories was analysed by a test of proportion ( $\mathrm{p} \leq \mathrm{0.01}$ ). Phytosociological descriptors were calculated for analysing the structure of the communities (Martins, 1993). The data ordination concerning the floristic-structural pattern among the communities was evaluated by the Non-metric multidimensional scaling (NMDS) method from the abundance matrix of the species in each sampled site. All analyses were performed using RStudio statistical software (R Development Core Team, 2015).

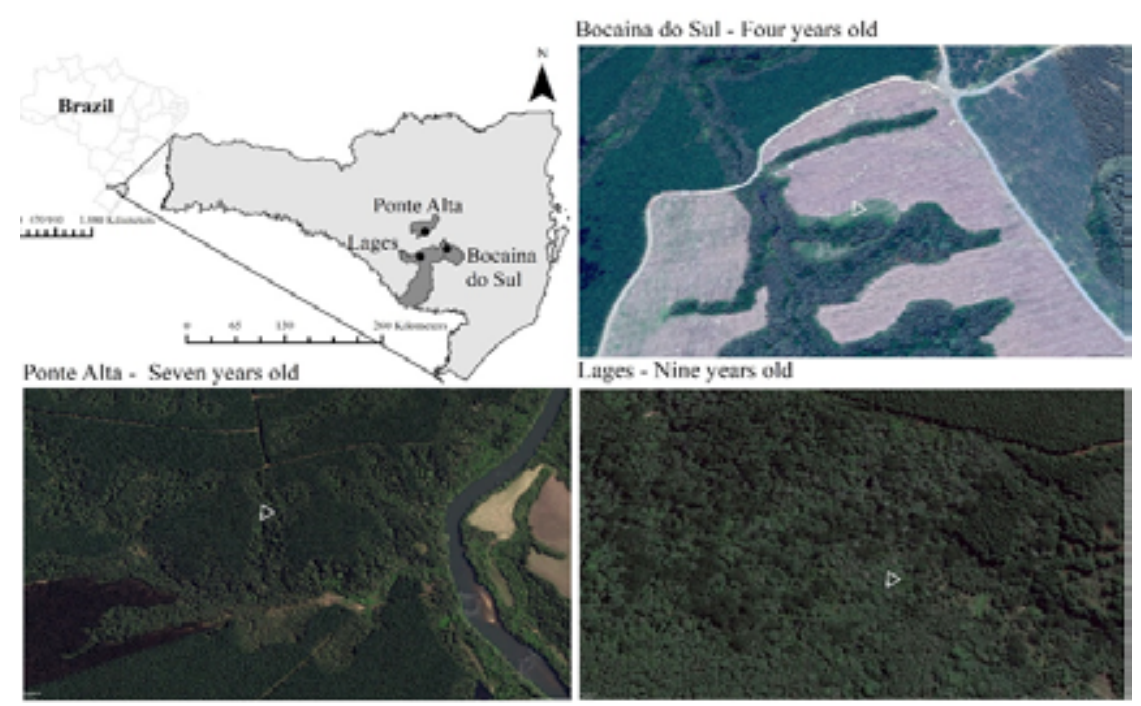

Figure 1. Location and landscape matrix of the study areas of the regenerating community in Mimosa scabrella Benth. understories located in Santa Catarina State South Plateau.

Source: Google Earth (2018). 


\section{RESULTS}

In the Mimosa scabrella understories, 2692 individuals belonging to 27 botanical families and 74 species were sampled. Of these, two were identified at the family level and three at the gender level. The ecological indexes evaluated in the three studied areas are presented in Table 2. The lowest Shannon diversity index $\left(\mathrm{H}^{\prime}\right)$ was recorded in the lesser understory (four years), presenting a significant difference from other sampled areas at a probability $<0.001$ (Hutcheson t-test). No significant differences were observed in the $\mathrm{H}^{\prime}$ value for the areas of seven and nine years.

Considering the three areas studied, the total number of individuals, families and species was proportional to the regeneration time of each $M$. scabrella understory, being higher in the nine-year understory (Table 2).

In the younger area (four years), Asteraceae was noted for its richness and abundance, representing approximately $27 \%$ of the species and $34 \%$ of the sampled individuals. In the seven-year understory, Lauraceae and Solanaceae represented approximately $30 \%$ of the species and individuals. In the older understory (nine years), Myrtaceae stood out as the richest (18\%) and most abundant (26\%) family.

In relation to the structural analysis, three species represented more than $50 \%$ of the importance value index (IVI) in the four years area, namely: Vernonanthura discolor (Spreng.) H.Rob., Croton reitzii L.B.Sm. \& Downs cf. and Baccharis uncinella DC. (Table 3).

Table 2. Regeneration characteristics in communities of Mimosa scabrella understories Benth. located in Santa Catarina State South Plateau.

\begin{tabular}{lccc}
\hline \multirow{2}{*}{ Ecological indicators } & \multicolumn{2}{c|}{ Mimosa scabrella Benth. populations } \\
\cline { 2 - 4 } Total number of individuals & Four years & Seven years & Nine years \\
\hline Number of species & 433 & 452 & 1807 \\
\hline Rarefied richness (433 individuals $\left.{ }^{*}\right)$ & 27 & 40 & 61 \\
Number of families & 27 & 39 & 39 \\
Shannon diversity index $\left(H^{\prime}\right)$ & 14 & 20 & 3.12 \\
\hline Pielou evenness index $\left(J^{\prime}\right)$ & 2.31 & 2.96 & 0.76 \\
\hline
\end{tabular}

* abundance limit for constructing the rarefaction curve using 1000 randomizations.

Table 3. List of tree species sampled in the Mimosa scabrella Benth. understory regenerating four, seven and nine years ago with their respective phytosociological descriptors and ecological groups.

\begin{tabular}{|c|c|c|c|c|c|c|c|}
\hline \multicolumn{8}{|c|}{ Mimosa scabrella Benth. understory in regeneration for four years } \\
\hline Family & Species & $\mathbf{N}$ & RD & RF & RDo & IVI & EG \\
\hline Euphorbiaceae & Croton reitzii L.B.Sm. \& Downs & 187 & 43.19 & 14.81 & 36.33 & 31.44 & PI \\
\hline Asteraceae & Baccharis uncinella DC. & 65 & 15.01 & 12.04 & 21.52 & 16.19 & PI \\
\hline Asteraceae & Vernonanthura discolor (Spreng.) H.Rob. & 64 & 14.78 & 11.11 & 9.56 & 11.82 & PI \\
\hline Asteraceae & Baccharis semiserrata DC. & 13 & 3 & 8.33 & 6.46 & 5.93 & PI \\
\hline Solanaceae & Solanum lacerdae Dusén & 19 & 4.39 & 6.48 & 5.57 & 5.48 & PI \\
\hline Lauraceae & Cinnamomum amoenum (Nees \& Mart.) Kosterm. & 22 & 5.08 & 4.63 & 1.83 & 3.85 & LS \\
\hline Sapindaceae & Matayba elaeagnoides Radlk. & 12 & 2.77 & 4.63 & 3.5 & 3.63 & LS \\
\hline Asteraceae & Piptocarpha angustifolia Dusén ex Malme & 6 & 1.39 & 3.7 & 3.36 & 2.82 & PI \\
\hline Solanaceae & Solanum variabile Mart. & 6 & 1.39 & 4.63 & 0.87 & 2.29 & PI \\
\hline Primulaceae & Myrsine coriacea (Sw.) R.Br. ex Roem. \& Schult. & 5 & 1.15 & 4.63 & 0.45 & 2.08 & ES \\
\hline Solanaceae & Solanum mauritianum Scop. & 3 & 0.69 & 2.78 & 1.6 & 1.69 & PI \\
\hline Fabaceae & Dalbergia frutescens (Vell.) Britton & 3 & 0.69 & 2.78 & 1.32 & 1.6 & ES \\
\hline Lauraceae & Nectandra lanceolata Nees & 6 & 1.39 & 1.85 & 0.61 & 1.28 & ST \\
\hline
\end{tabular}


Table 3. Continued...

\begin{tabular}{|c|c|c|c|c|c|c|c|}
\hline \multicolumn{8}{|c|}{ Mimosa scabrella Benth. understory in regeneration for four years } \\
\hline Family & Species & $\mathbf{N}$ & RD & RF & RDo & IVI & EG \\
\hline Aquifoliaceae & Ilex paraguariensis A.St.-Hil. & 1 & 0.23 & 0.93 & 2.3 & 1.15 & LS \\
\hline Lauraceae & Ocotea puberula (Rich.) Nees & 3 & 0.69 & 1.85 & 0.63 & 1.06 & ES \\
\hline Fabaceae & Inga lentiscifolia Benth. & 3 & 0.69 & 1.85 & 0.59 & 1.04 & PI \\
\hline Myrtaceae & Eugenia pluriflora DC. & 2 & 0.46 & 1.85 & 0.68 & 0.69 & ES \\
\hline Aquifoliaceae & Ilex sp. & 2 & 0.46 & 1.85 & 0.52 & 0.5 & UN \\
\hline Bignoniaceae & Jacaranda puberula Cham. & 2 & 0.46 & 1.85 & 0.47 & 0.5 & ES \\
\hline Clethraceae & Clethra scabra Pers. & 2 & 0.23 & 0.93 & 0.68 & 0.42 & PI \\
\hline Salicaceae & Casearia obliqua Spreng. & 1 & 0.23 & 0.93 & 0.35 & 0.42 & LS \\
\hline Aquifoliaceae & Ilex microdonta Reissek & 1 & 0.23 & 0.93 & 0.35 & 0.42 & LS \\
\hline Sapindaceae & Cupania vernalis Cambess. & 1 & 0.23 & 0.93 & 0.12 & 0.42 & ES \\
\hline Styracaceae & Styrax leprosus Hook. \& Arn. & 1 & 0.23 & 0.93 & 0.12 & 0.42 & ES \\
\hline Lauraceae & Cryptocarya aschersoniana $\mathrm{Mez}$ & 1 & 0.23 & 0.93 & 0.09 & 0.42 & LS \\
\hline Rosaceae & Prunus myrtifolia (L.) Urb. & 1 & 0.23 & 0.93 & 0.09 & 0.42 & ES \\
\hline Annonaceae & Annona rugulosa (Schltdl.) H.Rainer & 1 & 0.23 & 0.93 & 0.05 & 0.4 & LS \\
\hline Total & & 433 & 100 & 100 & 100 & 100 & \\
\hline \multicolumn{8}{|c|}{ Mimosa scabrella Benth. understory in regeneration for seven years } \\
\hline Family & Species & $\mathbf{N}$ & RD & RF & RDo & IVI & EG \\
\hline Clethraceae & Clethra scabra Pers. & 70 & 15.49 & 0.63 & 14.92 & 10.34 & PI \\
\hline Solanaceae & Solanum variabile Mart. & 49 & 10.84 & 0.63 & 17.18 & 9.55 & PI \\
\hline Lauraceae & Ocotea puberula (Rich.) Nees & 29 & 6.42 & 0.63 & 13.46 & 6.83 & ES \\
\hline Myrtaceae & Myrcia splendens (Sw.) DC. & 25 & 5.53 & 1.24 & 10.34 & 5.71 & ES \\
\hline Sapindaceae & Matayba elaeagnoides Radlk. & 40 & 8.85 & 0.63 & 6.94 & 5.47 & LS \\
\hline Dicksoniaceae & Dicksonia sellowiana Hook. & 46 & 10.18 & 1.25 & 0.82 & 4.08 & LS \\
\hline Primulaceae & Myrsine coriacea (Sw.) R.Br. ex Roem. \& Schult. & 26 & 5.75 & 3.13 & 2.9 & 3.92 & PI \\
\hline Aquifoliaceae & Ilex paraguariensis A.St.-Hil. & 27 & 5.97 & 0.63 & 4.88 & 3.83 & PI \\
\hline Lauraceae & Ocotea pulchella (Nees \& Mart.) Mez & 11 & 2.43 & 6.88 & 1.43 & 3.58 & ES \\
\hline Styracaceae & Styrax leprosus Hook. \& Arn. & 6 & 1.33 & 8.75 & 0.35 & 3.48 & ES \\
\hline Rosaceae & Prunus myrtifolia (L.) Urb & 21 & 4.65 & 3.13 & 1.84 & 3.2 & ES \\
\hline Melastomataceae & Miconia hyemalis A.St.-Hil. \& Naudin & 2 & 0.44 & 8.75 & 0.35 & 3.28 & PI \\
\hline Erythroxylaceae & Erythroxylum deciduum A.St.-Hil. & 1 & 0.22 & 8.13 & 0.05 & 2.8 & ES \\
\hline Asteraceae & Baccharis semiserrata DC. & 10 & 2.21 & 1.25 & 4.66 & 2.71 & PI \\
\hline Asteraceae & Vernonanthura discolor (Spreng.) H.Rob. & 11 & 2.43 & 1.25 & 4.12 & 2.6 & PI \\
\hline Fabaceae & Dalbergia frutescens (Vell.) Britton & 2 & 0.44 & 6.88 & 0.33 & 2.55 & ES \\
\hline Asteraceae & Baccharis uncinella DC. & 4 & 0.88 & 3.75 & 2.09 & 2.24 & PI \\
\hline Fabaceae & Inga lentiscifolia Benth. & 3 & 0.66 & 5.63 & 0.43 & 2.24 & LS \\
\hline Lauraceae & Cinnamomum amoenum (Nees \& Mart.) Kosterm. & 18 & 3.98 & 1.25 & 1.46 & 2.23 & LS \\
\hline Lauraceae & Nectandra grandiflora Nees & 3 & 0.66 & 4.38 & 0.65 & 1.9 & LS \\
\hline Lauraceae & Persea major (Meisn.) L.E.Kopp & 2 & 0.44 & 3.75 & 0.25 & 1.48 & LS \\
\hline Euphorbiaceae & Sapium glandulosum (L.) Morong & 2 & 0.44 & 3.13 & 0.58 & 1.38 & PI \\
\hline Canellaceae & Cinnamodendron dinisii Schwanke & 1 & 0.22 & 3.75 & 0.03 & 1.33 & ES \\
\hline Asteraceae & Piptocarpha angustifolia Dusén ex Malme & 6 & 1.33 & 0.63 & 2.01 & 1.32 & PI \\
\hline
\end{tabular}


Table 3. Continued...

\begin{tabular}{|c|c|c|c|c|c|c|c|}
\hline \multicolumn{8}{|c|}{ Mimosa scabrella Benth. understory in regeneration for seven years } \\
\hline Family & Species & $\mathbf{N}$ & RD & RF & RDo & IVI & EG \\
\hline Solanaceae & Solanum mauritianum Scop. & 3 & 0.66 & 0.63 & 2.39 & 1.23 & PI \\
\hline Euphorbiaceae & $\begin{array}{c}\text { Sebastiania commersoniana (Baill.) L.B.Sm. \& } \\
\text { Downs }\end{array}$ & 7 & 1.55 & 0.63 & 1.43 & 1.2 & ES \\
\hline Salicaceae & Casearia decandra Jacq. & 2 & 0.44 & 2.5 & 0.35 & 1.1 & LS \\
\hline Annonaceae & Annona rugulosa (Schltdl.) H.Rainer & 6 & 1.33 & 1.25 & 0.44 & 1.01 & LS \\
\hline Lauraceae & Nectandra lanceolata Nees & 5 & 1.11 & 1.25 & 0.48 & 0.94 & LS \\
\hline ceae & Araucaria angustifolia (Bertol.) Kuntze & 2 & 0.44 & 1.25 & 0.9 & 0.86 & PI \\
\hline Araucariaceae & Solanum lacerdae Dusén & 1 & 0.22 & 1.88 & 0.38 & 0.83 & PI \\
\hline Solanaceae & Solanum sanctaecatharinae Dunal. & 1 & 0.22 & 1.88 & 0.29 & 0.79 & ES \\
\hline Lauraceae & Nectandra megapotamica (Spreng.) Mez & 1 & 0.22 & 1.88 & 0.03 & 0.71 & LS \\
\hline Sapindaceae & $\begin{array}{l}\text { Allophylus edulis (A.St.-Hil. et al.) Hieron. ex } \\
\text { Niederl. }\end{array}$ & 2 & 0.44 & 1.25 & 0.37 & 0.69 & ES \\
\hline Lauraceae & Persea willdenovii Kosterm. & 1 & 0.22 & 1.25 & 0.35 & 0.61 & $\mathrm{UN}$ \\
\hline Anacardiaceae & Schinus terebinthifolius Raddi & 1 & 0.22 & 1.25 & 0.13 & 0.53 & PI \\
\hline Bignoniaceae & Jacaranda puberula Cham. & 1 & 0.22 & 1.25 & 0.02 & 0.5 & ES \\
\hline Aquifoliaceae & Ilex microdonta Reissek & 2 & 0.44 & 0.63 & 0.08 & 0.38 & LS \\
\hline Aquifoliaceae & Ilex dumosa Reissek & 1 & 0.22 & 0.63 & 0.17 & 0.34 & ES \\
\hline Myrtaceae & Eugenia pluriflora DC. & 1 & 0.22 & 0.63 & 0.16 & 0.33 & ES \\
\hline Total & & 452 & 100 & 100 & 100 & 100 & \\
\hline \multicolumn{8}{|c|}{ Mimosa scabrella Benth. understory in regeneration for nine years } \\
\hline Family & Species & $\mathbf{N}$ & RD & RF & RDo & IVI & EG \\
\hline Myrtaceae & Myrcia splendens (Sw.) DC. & 418 & 23.13 & 4.56 & 14.51 & 14.07 & ES \\
\hline Solanaceae & Solanum variabile Mart. & 155 & 8.58 & 4.56 & 14.73 & 9.29 & PI \\
\hline Asteraceae & Vernonanthura discolor (Spreng.) H.Rob. & 109 & 6.03 & 3.70 & 14.76 & 8.17 & PI \\
\hline Bignoniaceae & Jacaranda puberula Cham. & 111 & 6.14 & 4.56 & 11.36 & 7.35 & ES \\
\hline Primulaceae & Myrsine coriacea (Sw.) R.Br. ex Roem. \& Schult. & 202 & 11.18 & 4.27 & 3.88 & 6.44 & ES \\
\hline Asteraceae & Piptocarpha angustifolia Dusén ex Malme & 52 & 2.88 & 4.56 & 11.66 & 6.36 & PI \\
\hline Salicaceae & Casearia decandra Jacq. & 67 & 3.71 & 3.70 & 3.50 & 3.64 & LS \\
\hline Sapindaceae & Matayba elaeagnoides Radlk. & 61 & 3.38 & 4.56 & 2.36 & 3.43 & LS \\
\hline Sapindaceae & Cupania vernalis Cambess. & 55 & 3.04 & 3.99 & 1.91 & 2.98 & ES \\
\hline Primulaceae & Myrsine parvula (Mez) Otegui & 69 & 3.82 & 3.70 & 1.26 & 2.93 & ES \\
\hline Lauraceae & Nectandra lanceolata Nees & 59 & 3.27 & 3.42 & 1.61 & 2.77 & LS \\
\hline Proteaceae & Roupala montana Aubl. & 35 & 1.94 & 3.99 & 1.97 & 2.63 & LS \\
\hline Aquifoliaceae & Ilex paraguariensis A.St.-Hil. & 36 & 1.99 & 3.70 & 2.10 & 2.60 & PI \\
\hline Annonaceae & Annona rugulosa (Schltdl.) H.Rainer & 39 & 2.16 & 3.99 & 0.91 & 2.35 & LS \\
\hline Primulaceae & Myrsine umbellata Mart. & 53 & 2.93 & 3.13 & 0.80 & 2.29 & PI \\
\hline ceae & Araucaria angustifolia (Bertol.) Kuntze & 37 & 2.05 & 2.85 & 0.61 & 1.84 & PI \\
\hline Araucariaceae & Ilex brevicuspis Reissek. & 29 & 1.60 & 2.28 & 0.61 & 1.50 & LS \\
\hline Myrtaceae & Myrtaceae sp & 29 & 1.60 & 2.28 & 0.50 & 1.46 & UN \\
\hline Rutaceae & Zanthoxylum rhoifolium Lam. & 19 & 1.05 & 2.85 & 0.46 & 1.45 & PI \\
\hline Lauraceae & Ocotea pulchella (Nees \& Mart.) Mez & 19 & 1.05 & 1.99 & 1.29 & 1.45 & ES \\
\hline
\end{tabular}


Table 3. Continued...

\begin{tabular}{|c|c|c|c|c|c|c|c|}
\hline \multicolumn{8}{|c|}{ Mimosa scabrella Benth. understory in regeneration for nine years } \\
\hline Family & Species & $\mathbf{N}$ & RD & RF & RDo & IVI & EG \\
\hline Solanaceae & Solanaceae 1 & 14 & 0.77 & 2.28 & 0.98 & 1.35 & $\mathrm{UN}$ \\
\hline Lauraceae & Ocotea puberula (Rich.) Nees & 24 & 1.33 & 1.99 & 0.36 & 1.23 & ES \\
\hline Meliaceae & Cabralea canjerana (Vell.) Mart. & 11 & 0.61 & 1.99 & 0.61 & 1.07 & ES \\
\hline Fabaceae & Dalbergia frutescens (Vell.) Britton & 15 & 0.83 & 1.14 & 0.78 & 0.92 & ES \\
\hline Myrtaceae & Myrcia hatschbachii D. Legrand. & 9 & 0.50 & 1.42 & 0.51 & 0.81 & ES \\
\hline Myrtaceae & Myrcia hartwegiana (O.Berg) Kiaersk. & 3 & 0.17 & 0.85 & 0.81 & 0.61 & ES \\
\hline Salicaceae & Casearia obliqua Spreng. & 4 & 0.22 & 1.14 & 0.31 & 0.56 & LS \\
\hline Lauraceae & Nectandra megapotamica (Spreng.) Mez & 5 & 0.28 & 1.14 & 0.22 & 0.55 & LS \\
\hline Euphorbiaceae & Sapium glandulosum (L.) Morong & 3 & 0.17 & 0.85 & 0.52 & 0.51 & PI \\
\hline Aquifoliaceae & Ilex theezans Mart. ex Reissek & 3 & 0.17 & 0.85 & 0.39 & 0.47 & ES \\
\hline Styracaceae & Styrax leprosus Hook. \& Arn. & 6 & 0.33 & 0.57 & 0.35 & 0.42 & ES \\
\hline Cannabaceae & Celtis iguanaea (Jacq.) Sarg. & 4 & 0.22 & 0.85 & 0.13 & 0.40 & PI \\
\hline Myrtaceae & Campomanesia xanthocarpa Berg & 4 & 0.22 & 0.85 & 0.06 & 0.38 & ES \\
\hline Erythroxylacea & Erythroxylum deciduum A.St.-Hil. & 3 & 0.17 & 0.85 & 0.10 & 0.37 & ES \\
\hline Salicaceae & Casearia sylvestris $\mathrm{Sw}$. & 4 & 0.22 & 0.85 & 0.04 & 0.37 & PI \\
\hline Cardiopteridaceae & Citronella paniculata (Mart.) Howard. & 1 & 0.06 & 0.28 & 0.72 & 0.35 & ES \\
\hline Euphorbiaceae & $\begin{array}{c}\text { Sebastiania commersoniana (Baill.) L.B.Sm. \& } \\
\text { Downs }\end{array}$ & 4 & 0.22 & 0.57 & 0.14 & 0.31 & ES \\
\hline Canellaceae & Cinnamodendron dinisii Schwacke & 1 & 0.06 & 0.28 & 0.59 & 0.31 & PI \\
\hline Myrtaceae & Myrcia laruotteana Cambesse & 4 & 0.22 & 0.57 & 0.09 & 0.29 & ES \\
\hline Solanaceae & Solanum mauritianum Scop. & 2 & 0.11 & 0.28 & 0.40 & 0.26 & PI \\
\hline Clethraceae & Clethra scabra Pers. & 3 & 0.17 & 0.57 & 0.04 & 0.26 & PI \\
\hline Meliaceae & Cedrela fissilis Vell. & 3 & 0.17 & 0.57 & 0.02 & 0.25 & PI \\
\hline Sapindaceae & $\begin{array}{l}\text { Allophylus edulis (A.St.-Hil. et al.) } \\
\text { Hieron. ex Niederl. }\end{array}$ & 2 & 0.11 & 0.57 & 0.06 & 0.25 & ES \\
\hline Salicaceae & Xylosma ciliatifolia (Clos) Eichler & 1 & 0.06 & 0.28 & 0.39 & 0.24 & ES \\
\hline Sapindaceae & Allophylus guaraniticus (A. St.-Hil.) Radlk. & 2 & 0.11 & 0.57 & 0.04 & 0.24 & LS \\
\hline Rosaceae & Prunus myrtifolia (L.) Urb. & 2 & 0.11 & 0.57 & 0.03 & 0.24 & ES \\
\hline Solanaceae & Solanum sanctaecatharinae Dunal. & 2 & 0.11 & 0.57 & 0.02 & 0.23 & ES \\
\hline Aquifoliaceae & Ilex dumosa Reissek & 1 & 0.06 & 0.28 & 0.10 & 0.15 & LS \\
\hline Solanaceae & Solanum lacerdae Dusén & 1 & 0.06 & 0.28 & 0.09 & 0.14 & PI \\
\hline Fabaceae & Inga vera Willd. & 1 & 0.06 & 0.28 & 0.08 & 0.14 & LS \\
\hline Elaeocarpaceae & Sloanea hirsuta (Schott) Planch. ex Benth. & 1 & 0.06 & 0.28 & 0.05 & 0.13 & LS \\
\hline Myrtaceae & Calyptranthes concinna DC. & 1 & 0.06 & 0.28 & 0.05 & 0.13 & LS \\
\hline Myrtaceae & Myrciaria delicatula (DC.) O.Berg & 1 & 0.06 & 0.28 & 0.03 & 0.12 & LS \\
\hline Cardiopteridaceae & Citronella gongonha (Mart.) R.A.Howard & 1 & 0.06 & 0.28 & 0.02 & 0.12 & LS \\
\hline Myrtaceae & Eugenia pluriflora DC. & 1 & 0.06 & 0.28 & 0.02 & 0.12 & ES \\
\hline Aquifoliaceae & Ilex microdonta Reissek & 1 & 0.06 & 0.28 & 0.02 & 0.12 & LS \\
\hline Melastomataceae & Miconia sellowiana Naudim & 1 & 0.06 & 0.28 & 0.01 & 0.12 & UN \\
\hline Solanaceae & Solanum sp1 & 1 & 0.06 & 0.28 & 0.01 & 0.12 & UN \\
\hline Myrtaceae & Myrciaria sp. & 1 & 0.06 & 0.28 & 0.01 & 0.12 & UN \\
\hline
\end{tabular}




\begin{tabular}{lccccccc}
\hline \multicolumn{1}{c}{ Mimosa scabrella Benth. understory in regeneration for nine years } \\
\hline Family & Species & N & RD & RF & RDo & IVI & EG \\
Myrtaceae & Myrcia palustris DC. & 1 & 0.06 & 0.28 & 0.01 & 0.12 & ES \\
Rhamnaceae & Rhamnusphaerosperma Sw. & 1 & 0.06 & 0.28 & 0.00 & 0.11 & ES \\
\hline Total & & 1807 & 100 & 100 & 100 & 100 & \\
\hline
\end{tabular}

Legend: N: total number of individuals; RD: relative density (\%); RF: relative frequency (\%); RDo: relative dominance IVI: importance value index (\%); EG: ecological group; PI: pioneer; ES: early secondary; LS: late secondary (\%); UN: undetermined.

Among the species of higher IVI in the understory of seven years (Clethra scabra Pers., Solanum variabile Mart., Ocotea puberula (Rich.) Nees, Myrcia splendens (Sw.) DC. and Matayba elaeagnoides Radlk), three already belong to the secondary group (Table 3 ).

In the more advanced understory (nine years), the structure was represented by more than $50 \%$ of IVI by the following species: Myrcia splendens (Sw.) DC., S. variabile, $V$. discolor, Jacaranda puberula Cham., $M$. coriacea and Piptocarpha angustifolia (Table 3).

Regarding ecological group participation in the different succession times, it is observed that there is a significant difference by the test of proportions $(<0.001)$.
The most representative group in the understory with four years of natural regeneration was the pioneer group (85.2\%). For the environments in seven and nine years of regeneration, the early secondary group contributed with $24.1 \%$ and $53.7 \%$, respectively. The seven-year understory had the highest abundance of late secondary species $(28.4 \%)$.

The data ordination produced by the NMDS presented a stress value of 14.34 , indicating that the ordination is representative and adequate for the interpretation. From the ordination represented in the diagram (Figure 2), it was verified that the different understory ages of $M$. scabrella showed species substitution.

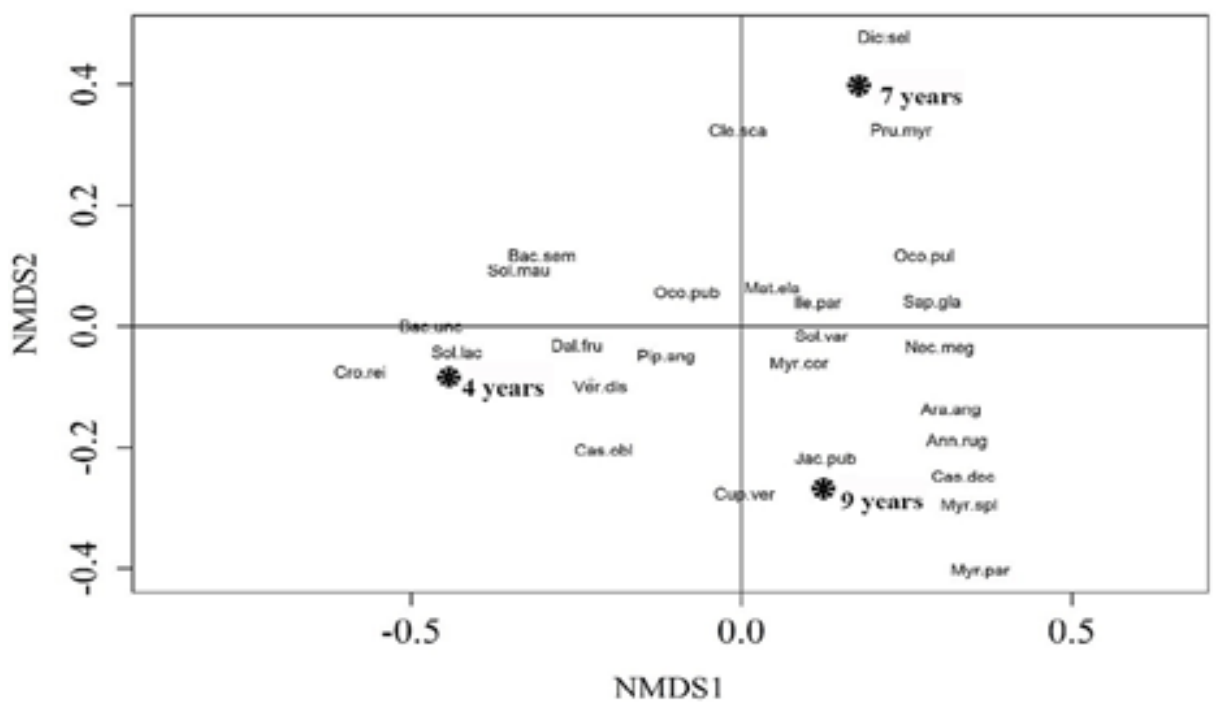

Figure 2. Ordination diagram produced by Non-metric Multidimensional Scaling analysis. (NMDS) of a regenerating tree community in Mimosa scabrella Benth. understories located in the Santa Catarina State South Plateau. Where: Ann.rug: Annona rugulosa; Ara.ang: angustifolia; Bac.sem: Baccharis semiserrata; Bac.unc: Baccharis uncinella; Cas.dec: Casearia decandra; Cas.obl: Casearia obliqua; Cle.sca: Clethra scabra; Cro.rei: Croton reitzii; Cup.ver: Cupania vernalis; Dal.fru: Dalbergia frutescens;Ile.par: Ilex paraguariensis; Jac.pub: Jacaranda puberula; Mat.ela: Matayba elaeagnoides; Myr.cor: Myrsine coriacea; Myr.par: Myrsine parvula; Myr.spl: Myrcia splendens; Nec.meg: Nectandra megapotamica; Oco.pub: Ocotea puberula; Oco.pul: Ocotea pulchella; Pip.ang: Piptocarpha angustifolia; Pru.myr: Prunus myrtifolia; Sap.gla: Sapium glandulosum; Sol.lac: Solanum lacerdae; Sol.mau: Solanum mauritianum; Sol.var: Solanum variabile; Ver.dis: Vernonanthura discolor. 


\section{DISCUSSION}

The lowest Shannon ( $\left.\mathrm{H}^{\prime}\right)$ diversity index value was recorded in the younger understory (four years), with this value being higher than that found by Barbosa et al. (2009) who studied an Araucaria angustifolia (Bertol.) Kuntze understory for about 12 years. The community registered in the areas of seven and nine years showed low dominance according to the Pielou index values (J'). These results corroborate with those found in regeneration studies carried out in Araucaria Forest (Narvaes et al., 2005; Kanieski et al., 2012).

The short temporal scale between the studied sites (understories of seven and nine years) can be evidenced when the rarefied richness is analyzed. Still, the environments structure is characterized by an increase in abundance and wealth, evidencing the successional advance.

Species belonging to Asteraceae and Solanaceae were prominent in the lower understory (four years), as they preferentially occur in open environments and fragment edges (Tabarelli \& Mantovani, 1999; Barroso \& Bueno, 2002). This result suggests that the successional process is still in its initial phase, unlike the other studied environments (seven and nine years), where there was greater Lauraceae and Myrtaceae representation. These families are commonly registered in Araucaria Forest studies at more advanced stages in Southern Brazil (Jarenkow \& Baptista, 1987; Narvaes et al., 2005; Herrera et al., 2009; Silva et al., 2012).

Based on the observation of the most representative species in the structural analysis in the area of four years, it was verified that they are pioneer species with characteristics of initial succession stages in Araucaria Forest, becoming abundant in altered environments (Barroso \& Bueno, 2002; Machado et al., 2006; Herrera et al., 2009; Ferreira et al., 2012). In the seven years understory, the species with higher IVI (Ocotea puberula (Rich.) Nees, Myrcia splendens (Sw.) DC. and Matayba elaeagnoides Radlk) belong to the secondary group. This aspect shows that although the area is in the initial stage due to the short regeneration time, it is possible to verify the species substitution dynamics in relation to the ecological succession.

Some pioneers are still present in the more advanced understory (nine years), where early and late secondary species participation represent more than half of the registered individuals. The species with higher IVI in these areas were: M. splendens (ES), S. variabile (PI) and $M$. coriacea (ES), and are zoochorically dispersed, demonstrating the presence of interactions with the fauna and of this ecosystem's functionality having returned.

The data ordination (NMDS) showed that the different ages of $M$. scabrella underwent species substitution, and this floristic-structural gradient is associated to the successional dynamics process. The predominance of species belonging to the pioneer ecological group is associated with the younger community (four years), as well as the higher occurrence of late secondary species in the seven and nine-year-old understories. Considering this aspect, it is possible to verify that $M$. scabrella presents as a potential facilitator species for altered areas, since the increase in diversity from the initial (four years) to medium (seven and nine years) can act in improving the soil and climatic conditions. In this way, it is possible to verify that, as the development of these populations occurs, the conditions for successional processes to occur can be expanded, enabling more species to be established. However, it is important to emphasize that this process will be conditioned by the presence of remnant fragments in the landscape, which directly act as propagule sources for the revegetation of these areas.

It is also worth noting the importance of preserving areas in the secondary succession process, since they are vegetation cover with potentially endangered species, such as Araucaria angustifolia and Cedrela fissilis, recorded in this study and included on the threatened species list of the International Union for Conservation of Nature and Natural Resources (IUCN, 2016).

\section{CONCLUSIONS}

The highest regenerant richness was recorded in the Mimosa scabrella understory at a more advanced age.

The areas under natural regeneration in Mimosa scabrella understories with different ages presented different floristic-structural patterns, which are compatible with the characteristic trends of successional dynamics of Araucaria Forest, mainly due to the effect of the landscape matrix.

The increase in diversity in the understories of the Mimosa scabrella populations in the early to middle stages suggests the potential of this species as a facilitator for restoring altered areas. 


\section{ACKNOWLEDGMENTS}

The authors would like to thank Santa Catarina State University, the Maintenance Support Fund and the Development of Higher Education in Santa Catarina for the granting scholarships to Ferreira and Gomes, to the Coordination for the Improvement of Higher Education Personnel for granting the scholarship to Stedille, the Foundation for Support of Research and Innovation of Santa Catarina and Klabin S/A.

\section{SUBMISSION STATUS}

Received: 31 Jan., 2017

Accepted: 30 June, 2018

\section{CORRESPONDENCE TO}

\section{Lilian Iara Stedille}

Universidade do Estado de Santa Catarina, Av. Luiz de Camões, 2.090, CEP 88520-000, Lages, SC, Brasil e-mail: lilian.stedille@gmail.com

\section{REFERENCES}

Alvarenga AP, Botelho SA, Pereira IM. Avaliação da regeneração natural na recomposição de matas ciliares em nascentes na região Sul de Minas Gerais. Cerne 2006; 12(4): 360-372. 10.5902/19805098403

Alvares CA, Luiz SJ, Sentelhas PC, Gonçalves JLM, Sparovek G. Köppen's climate classification map for Brazil. Meteorologische Zeitschrift 2014; 22(6): 711-728. 10.1127/0941-2948/2013/0507

Barbosa CEA, Benato T, Cavalheiro AL, Torezan JMD. Diversity of regenerating plants in reforestations with angustifolia (Bertol.) O. Kuntze of 12, 22, 35, and 43 years of age in Paraná state, Brazil. Restoration Ecology 2009; 17(1): 60-67. 10.1111/j.1526-100X.2007.00335.x

Barroso GM, Bueno OL. Compostas. In:ReitzR. Flora ilustrada catarinense. Itajaí: Herbário Barbosa Rodrigues; 2002.

Budowski GN. Distribution of tropical American rain forest species in the light of sucessional processes. Turrialba 1965; 15: 40-42.

Callaway RM. Positive interactions among plants. The Botanical Review 1995; 61(4): 306-349. 10.1007/BF02912621

Chada SS, Campello EFC, Faria SM. Sucessão vegetal em uma encosta reflorestada com leguminosas arbóreas em Angra dos Reis, RJ. Revista Árvore 2004; 28(6): 801-809. 10.1590/S0100-67622004000600005
Dutra VF, Amorim MP. Mimosa scabrella Benth. 2012 [cited 2013 May 11]. Available from: https://bit.ly/2yIoUMo

Flora do Brasil 2020 [Internet]. Lista de espécies da flora do Brasil. 2019 [cited 2019 May 20]. Available from: http://floradobrasil.jbrj.gov.br

Haggar J, Wightman K, Fisher R. The potential of plantations to foster woody regeneration within a deforested landscape in lowland Costa Rica. Forest Ecology and Management 1997; 99: 55-64. 10.1016/S0378-1127(97)00194-1

Herrera HAR, Rosot NC, Rosot MAD, Oliveira EB. Análise florística e fitossociológica do componente arbóreo da Floresta Ombrófila Mista presente na reserva florestal EMBRAPA/EPAGRI, Caçador, SC - Brasil. Revista Floresta 2009; 39(3): 485-500. 10.5380/rf.v39i3.15349

Instituto Brasileiro de Geografia e Estatística - IBGE. Manual técnico da vegetação brasileira. 2. ed. Rio de Janeiro: IBGE; 2012.

Inoue MT, Roderjan CV, Kuniyoshi YS. Projeto madeira do Paraná. Curitiba: Fundação de Pesquisas Florestais do Paraná; 1984.

International Union for Conservation of Nature - IUCN. The IUCN red list of threatened species. [cited 2016 June 16]. Available from: www.iucnredlist.org.

Jarenkow JA, Batista LRM. Composição florística e estrutura da Mata com Araucária na Estação Ecológica de Aracuri, Esmeralda, RS. Napaea 1987; 3: 9-18.

Kabakoff RP, Chazdon RL. Effects of canopy species dominance on understorey light avilability in low-elevation secondary forest stands in Costa Rica. Journal of Tropical Ecology 1996; 12(6): 779-788. 10.1017/S0266467400010038

Kanieski MR, Longhi SJ, Narvaes IS, Soares PRC, LonghiSantos T, Callegaro RM. Diversidade e padrões de distribuição espacial de espécies no estágio de regeneração natural em São Francisco de Paula, RS, Brasil. Revista Floresta 2012; 42(3): 509-518. 10.5380/rf.v42i3.25037

Klein RM. Ecologia da flora e vegetação do Vale do Itajaí. Revista Sellowia 1980; 32: 164-369.

Martins FR. Estrutura de uma floresta mesófila. São Paulo: Editora Unicamp; 1993.

Melo ACG, Durigan G. Evolução estrutural de reflorestamentos de restauração de matas ciliares no Médio Vale do Paranapanema. Scientia Forestalis 2007 [cited 2019 July 3]; 73:101-111. Available from: https://bit.ly/2YxYWqe

Narvaes IS, Brena DA, Longhi SJ. Estrutura da regeneração natural em Floresta Ombrófila Mista na Floresta Nacional de São Francisco de Paula, RS. Ciência Florestal 2005; 15(4): 331-342. 10.5902/198050981871

R Development Core Team. The R Project for Statistical Computing [software]. 2015 [cited 2014 Mar. 1]. Available from: http://www.R-project.org.

Reis A, Kageyama PY. Restauração de áreas degradadas utilizando interações interespecíficas. In: Kageyama PY, 
Oliveira RE, Reitz R, Klein R, Reis A. Projeto Madeira de Santa Catarina: levantamento das espécies florestais nativas em Santa Catarina com a possibilidade de incremento e desenvolvimento. Itajaí: Herbário Barbosa Rodrigues; 1978.

Silva AC, Higuchi P, Aguiar MD, Negrini M, Neto JF, Hess AF. Relações florísticas e fitossociologia de uma Floresta Ombrófila Mista Montana secundária em Lages, SC. Ciência Florestal 2012; 22(1):193-206. 10.5902/198050985091
Santos SC, Budke JC, Muller A. Regeneração de espécies arbóreas sob a influência de Merostachys multiramea Hack. (Poaceae) em uma floresta subtropical. Acta Botanica Brasilica 2012; 26(1): 218-229. 10.1590/S0102-33062012000100021

Tabarelli M, Mantovani W. Clareiras naturais e a riqueza de espécies pioneiras em uma Floresta Atlântica Montana. Revista Brasileira de Biologia 1999; 59: 251-261. 10.1590/S0034-71081999000200009 